\title{
Remarques sur le comportement du neptunium 237 dans le milieu et les chaînes alimentaires
}

\author{
R. BITTEL $\left(^{\star}\right)$ \\ (Manuscrit reçu le 21 juin 1982)
}

RÉSUMÉ

L'objet de ce document est de faire le point, à l'aide de références récentes, sur les risques dus aux rejets et au stockage des déchets de ${ }^{237} \mathrm{~Np}$. Cette recherche doit déboucher sur une étude plus large des comportements comparés des divers transuraniens et plus spécifiquement sur celle du ${ }^{237} \mathrm{~Np}$, quant à son absorption intestinale. II semble, sur la base d'expériences déjà anciennes, que la toxicité de ce radionucléide soit, à activités ingérées égales, supérieure à celle des autres transuraniens, en raison d'un facteur de transfert intestinal, plus élevé, qui pourrait être sujet à révision.

\section{ABSTRACT}

A review has been made of the latest literature on the hazards from abnormal releases of ${ }^{237} \mathrm{~Np}$ with a view to opening on to a broader study of the compared behaviours of various transuranic elements and more especially the intestinal absorption of ${ }^{237} \mathrm{~Np}$. It appears at the light of experimental data that, for the same ingested activities, neptunium has a higher toxicity than the other transuranic elements because of a higher intestinal transfer factor, which might be revised.

\section{INTRODUCTION}

Parmi les divers isotopes du neptunium, on n'envisage ici que l'isotope dont la période est la plus longue, ${ }^{237} \mathrm{~Np}$. II se forme à partir de ${ }^{235 \mathrm{U}}$ et de $238 \mathrm{U}$. Dans les réacteurs à uranium naturel ou peu enrichi, la voie dominante est la capture unique d'un neutron par $238 \mathrm{U}$ qui, par réaction $(\mathrm{n}, 2 \mathrm{n})$, produit ${ }^{237} \mathrm{U}$ et ${ }^{237} \mathrm{~Np}$ par décroissance $\beta$. Dans les réacteurs à uranium fortement enrichi, des captures successives de neutrons par $235 \mathrm{U}$ conduisent également à $237 \mathrm{U}$ et à ${ }^{237} \mathrm{~Np} .{ }^{237} \mathrm{~Np}$ se forme aussi par décroissance $\alpha$ à partir de ${ }^{241} \mathrm{Am}[2,15,23]$. Sous irradiation neutronique, ${ }^{237} \mathrm{~Np}$ donne ${ }^{238} \mathrm{~Np}$ puis $238 \mathrm{Pu}$; ce dernier, émetteur alpha de grande activité massique, est utilisé dans les stimulateurs cardiaques. ${ }^{237} \mathrm{~Np}$ est présent dans

(*) Commissariat à l'énergie atomique, IPSN, Département de Protection, SPS/LPEP, BP 6. 92260 Fontenay-aux-Roses. 
les combustibles nucléaires irradiés mais à des activités massiques beaucoup plus faibles que celles des plutoniums, américiums et curiums [23]. Au moment de son déchargement d'un réacteur à eau légère de type PWR, une tonne de combustible contient environ $35 \mathrm{~kg}$ de produits de fission et $10 \mathrm{~kg}$ de transuraniens. Dans les déchets de haute activité, ${ }^{237} \mathrm{~Np}$ n'a, du fait de son activité, d'importance relative qu'après un très grand laps de temps $[2,7,10,12,13,14,20]$.

Des études récentes montrent que ${ }^{237} \mathrm{~Np}$ est mieux absorbé par voie gastro-intestinale que les autres transuraniens et il semble qu'il s'agisse d'un effet de masse (cf. VI) : cela donne une importance nouvelle à l'examen des données relatives à cet élément $[7,12,37,38]$ et le long cheminement des recherches concernant le recyclage des transuraniens contenus dans les combustibles irradiés [17] ne peut faire oublier que ${ }^{237} \mathrm{~Np}$ pose et posera encore longtemps un problème actuel et persistant.

\section{CARACTÉRISTIQUES RADIO-PHYSICO-CHIMIQUES}

Paŕ un certain nombre d'aspects, le neptunium s'écarte des autres uranides et du curium et se rapproche d'éléments très éloignés dans la classification périodique : alcalins et alcalino-terreux en particulier. II faut d'abord souligner que le neptunium se comporte très différemment suivant qu'il se trouve inclus dans des combinaisons solides ou bien qu'il est en solution.

Dans les solides, $\mathrm{Np}$ est stable à l'état tétravalent sous forme d'oxyde $\mathrm{NpO}_{2}$, homologue de $\mathrm{UO}_{2}$, de $\mathrm{PuO}_{2}$ et d'autres oxydes de terres rares tétravalentes avec lesquels il réalise des solutions solides: le comportement physicochimique est imposé par l'oxyde dominant (notamment en ce qui concerne la solubilité "apparente"), par les traitements physiques subis (pression, température) et par la présence de complexants.

En solution, la forme ionique la plus stable est le cation complexe monovalent neptunyl $\mathrm{NpO}_{2}{ }^{+}$correspondant à la valence $\mathrm{V}$. $\mathrm{NpO}_{2}{ }^{+}$est, vis-à-vis des autres transuraniens, caractérisé par une hydrolyse faible et une dismutation nulle dans une zone de $\mathrm{pH}$ très large englobant les $\mathrm{pH}$ rencontrés dans les milieux physiques et dans les organismes vivants. La tendance à la complexation du neptunium est comparable à celle des ions alcalins et alcalino-terreux et de l'ion $\mathrm{UO}_{2}{ }^{++}$. La stabilité des complexes carboniques est faible mais leur existence semble possible en présence de concentrations non négligeables en $\mathrm{NpO}_{2}{ }^{+}$et de concentrations très élevées en ions bicarboniques [3].

L'activité massique de ${ }^{237} \mathrm{~Np}$ est égale à 26 G.Bq. $\mathrm{kg}^{-1}$ [20]. Elle est 90 fois inférieure à celle de ${ }^{239} \mathrm{Pu}$ et 25000 fois à celle de $238 \mathrm{Pu}$. Cependant, la radiolyse en solution n'est pas négligeable et ramène les composés dissous à l'état de $\mathrm{Np}(\mathrm{V})$, sous forme de l'ion monovalent neptunyl $\mathrm{NpO}_{2}{ }^{+}$(autoréduction alpha de $\mathrm{Np}(\mathrm{VI})$ à une vitesse égale à $3,1 \pm 0,2.10^{-9} \mathrm{~s}^{-1}$ et avec un rendement radiochimique $\mathrm{G}$ de 6,4 ions par $100 \mathrm{eV}$ [ [5].

Les dimensions ioniques de $\mathrm{Np}$, comme d'ailleurs celles de Am et $\mathrm{Cm}$ sont voisines de celles de $\mathrm{Ca}^{++}$et $\mathrm{Na}^{+}$, ce qui peut permettre de prévoir les phénomènes de sorptions des ions en solution correspondants sur et dans les minéraux argileux $[11,24]$. 


\section{NEPTUNIUM ET SOLS, SOUS-SOLS ET FORMATIONS GÉOLOGIQUES}

II y a trois problèmes essentiels :

- celui qui concerne les micro-organismes des sols et des sous-sols, et des gaines rhizosphériques;

- la mobilité de l'ion neptunyl;

- les transferts sols $\rightarrow$ plantes qui seront évoqués ultérieurement.

\section{III.1. Incidence des micro-organismes des sols}

Les micro-organismes des sols ont, en général, une grande incidence sur le comportement de l'uranium et des transuraniens [27, 40, 41] :

- modification de la forme physico-chimique ;

- modification de l'absorption racinaire.

Pour que les micro-organismes jouent ces rôles, il faut évidemment qu'ils résistent aux phénomènes de toxicité impliqués par la présence des activités locales relativement plus élevées en certains points des sols (rhizosphère) et des formations géologiques. Ces recherches ne sont qu'à leur début mais, dès maintenant, elles peuvent contribuer à prévoir les conséquences de rejets contrôlés de transuraniens. II suffit de rappeler ici l'incidence de la biogéochimie microbienne dans la connaissance et dans la compréhension du cycle de l'uranium dans l'environnement [41].

\section{III.2. Mobilité du neptunium [2, 4, 22, 26, 28, 30, 31]}

Des expérimentations in vitro ont montré que l'ion neptunyl $\mathrm{NpO}_{2}{ }^{+}$était, relativement aux autres transuraniens, très mobile dans les sols, les soussols et les formations géologiques perméables à l'eau. La mobilité de $\mathrm{NpO}_{2}{ }^{+}$ augmente quand le $\mathrm{pH}$ diminue, sans que la structure des colloïdes argileux et humiques soit affectée (ce qui oppose $\mathrm{Np}$ à $\mathrm{Am}$ et $\mathrm{Cm}$ ). Le neptunium IV formé par réduction in situ est très fortement "sorbé" par les argiles et les minéraux associés dans les sols. Des expérimentations ont montré que I'ion $\mathrm{NpO}_{2}{ }^{+}$se comporte presque comme les ions alcalins et alcalino-terreux, notamment avec les argiles. La présence dans les sols de matières humiques diminue la mobilité de $237 \mathrm{~Np}$. Mais ce sont les particules les plus fines des argiles qui, in situ, sont le plus souvent responsables de la "sorption" et des phénomènes de retard de migration. Le rapprochement qu'on peut faire entre le comportement des ions $\mathrm{NpO}_{2}{ }^{+}$et $\mathrm{Na}^{+}$conduit à attacher une attention particulière aux échanges d'ions $\mathrm{NpO}_{2}{ }^{+} / \mathrm{Na}^{+}$: les $\mathrm{K}_{d}$ (1) de $\mathrm{NpO}_{2}{ }^{+}$sont inversement proportionnels aux concentrations en $\mathrm{Na}^{+}$dans la phase liquide. Expérimentalement, il a été constaté que les $k_{d}$ pour ${ }^{237} \mathrm{~Np}$ étaient compris entre ceux de ${ }^{99} \mathrm{Tc}$ (probablement anionique) et ceux de ${ }^{241} \mathrm{Am}^{3+}$. Les facteurs de retardement (2) sont compris entre ceux de ${ }^{00} \mathrm{Sr}^{2+}$ et ceux de $137 \mathrm{Cs}^{+}$et sont très inférieurs à ceux des plutoniums et des américiums $\left({ }^{99} \mathrm{TcO}_{4}{ }^{-}: \mathrm{R}=1 ;{ }^{90} \mathrm{Sr}: \mathrm{R}=43 ;{ }^{237} \mathrm{~Np}: \mathrm{R}=250 ;{ }^{137} \mathrm{Cs}: \mathrm{R}=700\right.$; $\left.237 \mathrm{Pu}: \mathrm{R}=1100 ; 241 \mathrm{Am}: \mathrm{R}=10^{5}\right)$. Sur des argiles du type illite, à $\mathrm{pH}=6$, les valeurs expérimentales des $\mathrm{k}_{\mathrm{d}}$ ont été les suivantes (en I. $\left.\mathrm{kg}^{-1}\right)$ : $\mathrm{Sr}^{++}$et $\mathrm{NpO}_{2}^{+}: 5.10^{1} ; \mathrm{Cs}^{+}: 10^{3} ; \mathrm{Am}^{3+}$ et $\mathrm{Pu}$ (IV) : $10^{4}$ [26].

\footnotetext{
(1) $k_{d}=\frac{\text { activité massique de la phase solide }}{\text { activité massique de la phase liquide }}$ (équilibre) (en I. $\left.\mathrm{kg}^{-1}\right)$.

(2) Facteur de retardement $=\frac{\text { migration de }{ }^{237} \mathrm{~Np}\left(\mathrm{~cm} \cdot \mathrm{s}^{-1}\right)}{\text { migration de l'eau }\left(\mathrm{cm} \cdot \mathrm{s}^{-1}\right)}$ (sans unité).
} 
La rétention relativement faible de $237 \mathrm{NpO}_{2}{ }^{+}$par les argiles conduit à un lessivage assez important dans les sols, les facteurs s'opposant à ce lessivage étant surtout les matières organiques et de fortes teneurs en argile. Les modèles valables pour la migration des ions alcalino-terreux, et en particulier pour ${ }^{90} \mathrm{Sr}$, semblent applicables pour ${ }^{237} \mathrm{~Np}$, en première approximation.

\section{ABSORPTION RACINAIRE [29, 33, 34, 35]}

L'expérimentation montre que les facteurs de transfert $\mathrm{F}$ sol/plantes (1) sont, pour des conditions comparables, fortement supérieurs à ceux des autres transuraniens. II semble que les légumineuses prélèvent plus de neptunium que les graminées (comme c'est le cas des alcalino-terreux). Après une contamination unique du sol, les différentes coupes de luzerne garde le même niveau de $\mathrm{Np}$ (comme c'est d'ailleurs le cas pour les autres transuraniens).

La mobilité relative élevée du $\mathrm{Np}$ chez les végétaux a été mise en évidence par les mesures effectuées sur les grains de blé entiers et décortiqués. Les enveloppes sont plus contaminées que le grain, mais le rapport des activités balles / grain est plus faible d'un facteur 10 pour $\mathrm{Np}$ que pour $\mathrm{Pu}$ et $\mathrm{Cm}$. En ce qui concerne la paille et le grain total, les différences sont encore plus grandes: Np apparaît donc comme le transuranien le plus mobile après incorporation dans la plante.

\section{NEPTUNIUM ET MILIEU AQUATIQUE}

Les données relatives au prélèvement de $\mathrm{NpO}_{2}{ }^{+}$par les organismes aquatiques sont rares. II semble que l'accurnulation soit lente et faible et, surtout, qu'elle soit indépendante de la température. II s'agirait donc d'une absorption physique, sans que le métabolisme des animaux intervienne. Les périodes biologiques ont cependant été définies chez quelques espèces marines ; à titre indicatif $[9,25]$ :

Crevette $\quad T_{b 1}=4 j$ (période biologique courte).

$\mathrm{T}_{\mathrm{b} 2}=252 \mathrm{j}$ (période biologique longue).

Moule $\quad \mathrm{T}_{\mathrm{b} 2}=331 \mathrm{j}$ (période biologique longue).

Chez l'oursin Echinus esculentus prélevé au voisinage du Centre de Windscale les rapports:

sont de l'ordre de 10.

$\frac{{ }^{239} \mathrm{Pu} \text { tissus }}{237 \mathrm{~Np} \text { tissus }} / \frac{239 \mathrm{Pu} \text { déchargé }}{237 \mathrm{~Np} \text { déchargé }}$

II faut cependant signaler que l'algue Fucus serratus accumule plus Np que $\mathrm{Pu}$.

Lors du traitement des eaux en vue de leur potabilité, Np suit le sort des alcalins [8] (on n'élimine qu'une faible proportion des ions $\mathrm{NpO}_{2}{ }^{+}$présents).

(1) $\mathrm{F}=\frac{\text { activité massique de la plante (activité } \mathrm{g}^{-1} \text { ) }}{\text { activité massique du sol (activité } \mathrm{g}^{-1} \text { ) }}$ (sans unité). 


\section{MÉTABOLISME CHEZ LES ANIMAUX SUPÉRIEURS}

L'absorption intestinale du neptunium "soluble" est nettement plus élevée que celle des plutoniums et transplutoniens : chez le rat, elle est de l'ordre de 1 pour 100,100 fois supérieure à celle de $238 \mathrm{Pu}$ et de ${ }^{241} \mathrm{Am}, 25$ fois environ supérieure à celle de ${ }^{244} \mathrm{Cm}$ [26]. II semble que, contrairement à ce qu'on constate pour les autres actinides, ${ }^{237} \mathrm{~Np}$ lié à la matière organique des aliments soit moins bien incorporé que ${ }^{237} \mathrm{~Np}$ des combinaisons minérales [37].

La toxicité chimique du neptunium est élevée : chez le mouton par exemple, la dose létale $100 / 100$ en 3 jours est de l'ordre de $12 \mathrm{mg}$ par $\mathrm{kg}\left(310 \mathrm{~Bq} . \mathrm{g}^{-1}\right)$. Après injection de $1,5 \mathrm{mg}$ par $\mathrm{kg}$ [26], des symptômes cliniques et anatomopathologiques apparaissent déjà [20]. Cette toxicité rapproche $\mathrm{Np}$ des métaux lourds. En ce qui concerne la radiotoxicité [18, 19] $\mathrm{Np}(\mathrm{V})$ a un comportement assez voisin des alcalino-terreux ( ${ }^{\circ 0} \mathrm{Sr}$ par exemple) et s'éloigne des autres transuraniens. L'absorption intestinale élevée de ${ }^{237} \mathrm{~Np}\left(f_{1}=10^{-2}\right)$ semble en relation directe avec "un effet de masse". C'est, en particulier, ce qui paraît résulter des comparaisons entre les absorptions intestinales de radioisotopes différents du neptunium et de masses ingérées différentes de ${ }^{237} \mathrm{~Np}[7,37,38,39]$. En doublant les masses ingérées de ${ }^{237} \mathrm{~Np}$, on double la proportion assimilée au niveau du TGI On observe le contraire pour les radionucléides à forte activité massique. II semble qu'en ce qui concerne ${ }^{237} \mathrm{~Np}$ ingéré à forte activité globale, l'absorption intestinale soit accrue en raison de toxicité chimique. Si pour ${ }^{237} \mathrm{~Np}$, on admet $f_{1}=10^{-2}$, on prend pour Pu (sauf les oxydes) $f_{1}=10^{-4}$, et pour ${ }^{241} \mathrm{Am}-{ }^{244} \mathrm{Cm} \mathrm{f}_{1}=5.10^{-4}$ [12].

Le taux de transfert par unité de temps de Np du plasma au lait chez la brebis est faible, en comparaison avec celui de ${ }^{45} \mathrm{Ca}$ et ceux des autres transuraniens (tous les éléments sont injectés à l'état de chlorure) [16].

II convient donc ici de souligner que le modèle métabolique proposé par la CIPR 30 fait du neptunium 237 le transuranien le plus toxique, la limite d'incorporation par voie orale étant la plus faible en raison de la valeur élevée du facteur de transfert $f_{1}\left(10^{-2}\right)$.

\section{CONCLUSION}

Cette brève étude aide à mieux situer le neptunium 237 parmi les "toxiques nucléaires". Dans le milieu physique, l'ion neptunyl $\mathrm{NpO}_{2}{ }^{+}$, monovalent, a un comportement qui le rapproche des ions alcalins et alcalino-terreux et de l'ion uranyl $\mathrm{UO}_{2}{ }^{++}$. Sa mobilité pourrait conférer à cet ion un facteur de risque important dans le cas de rejets ou de défaillance dans les barrières géologiques des déchets radioactifs.

Dans les organismes, le neptunium présente une toxicité chimique importante lorsque la masse incorporée est grande et masque ainsi la radiotoxicité.

$\mathrm{Si}$, par voie respiratoire, la radiotoxicité paraît être du même ordre de grandeur que celles des autres transuraniens, par voie digestive, elle semble nettement supérieure ( $f_{1}$ plus grand), jusqu'à ce que des expériences menées actuellement dans les laboratoires spécialisés apportent éventuellement la preuve du contraire. 
R. BITTEL

Cette étude devrait se poursuivre par une recherche plus approfondie du neptunium 237, de l'américium 241 et du curium 244 dans les différents vecteurs alimentaires.

\section{RÉFÉRENCES BIBLIOGRAPHIQUES}

[1] BAES III C.F., SHARP R.D. Predicting radionuclide leaching from root zone soil for assessment applications. Trans. Americ. Nucl. Soc., 1981, 38, 111-112.

[2] BARTHELLIER A. Les éléments transuraniens dans le retraitement des combustibles nucléaires. Bull. Inform. Sci. Tech. (CEA), 1976, 217, 7-16.

[3] BILLON A. Sur l'existence des complexes carbonatés de $\mathrm{Np}$ (V) en solution aqueuse. In : AIEA. - Techniques for identifying transuranium speciation in aquatic environment, Ispra, March 1980. Vienne AIEA, 1981, 65-72.

[4] BONDIETTI E.A., TAMURA T. Physicochemical associations of plutonium and other actinides in soil. In : Transuranic elements in the environment (HANSON W.C., Ed). DOE/TIC - 22800, 1980, 145-164.

[5] BURNEY G.A., HARBOUR R.W. Radiochemistry of neptunium. NAS - NS 3080, 1974.

[6] CASEY H.W., MCCLELLAN R.O., CLARKE W.J., BUSTAD L.K. Acute toxicity of neptunium 237 and its relationship to liver function of sheep. Health Phys., 1963, 9, 827-834.

[7] COHEN B.L. Effects of ICRP Publication 30 and the 1980 BEIR report on hazard assessments of high - level waste. Health Phys., 1982, 42, 133-143.

[8] COREY J.C., BONI A.L. Removal of plutonium from drinking water by community treatment facilities, In : AIEA. - Transuranium nuclides in the environment, San Francisco, 17-21 Nov. 1975. Vienne, AIEA, 1976, 401-408.

[9] GUARY J.C., FOWLER S.W. Biokinetics of neptunium 237 in mussels and shrimps. Mar. Sci. Comm., 1977, 3, 211-229.

[10] GUILLAUME B. Problèmes posés par la présence d'éléments transuraniens dans les déchets du retraitement des combustibles nucléaires. Bull. Inform. Sci. Tech. (CEA), 1976, 217, 31-46.

[11] HAISSINSKY M., ADLOFF J.P. Dictionnaire radiochimique des éléments. Genève, SADESI, 1968.

[12] International commission on radiological protection (ICRP). Limits for intakes of radionuclides by workers. Metabolic data for neptunium, ICRP publication 30 , pt 2. Oxford: Pergamon press, 1980, 69-71.

[13] JENSEN B.K. The geochemistry of radionuclides with long half lives. Rapport RIS $\emptyset$ R-430, 1980.

[14] KREITER M.R., MENDEL J.E., MCKEE R.W. Transuranic wastes from the commercial light-water reactor cycle. In: Transuranic elements in the environment (HANSON W.C., Ed), DOE/TIC - 22800, 1980, 92-106.

[15] MADIC C., KOEHLY G. Les séparations chimiques des éléments transuraniens; Bull. Inform. Sci. Techn. (CEA), 1976, 217, 17-30.

[16] McCLELLAN R.O., CASEY H.W., BUSTAD L.K. Transfer of some transuranic elements to milk. Health Phys., 1962, 8, 689-694.

[17] McKAY H.A.C. Elimination des actinides présents dans les déchets par recyclage dans des réacteurs nucléaires. AIEA Bull., 1981, 23, 46-49.

[18] MORIN M. NENOT J.C., LAFUMA J. The behaviour of ${ }^{237} \mathrm{~Np}$ in the rat. Health Phys., 1973, 24, 311-315. 
[19] NENOT J.C., MASSE R., MORIN M., LAFUMA J. An experimental comparative study of the behaviour of ${ }^{237} \mathrm{~Np},{ }^{239} \mathrm{Pu},{ }^{241} \mathrm{Am}$ and ${ }^{242} \mathrm{Cm}$ in bone. Health Phys., $1972,22,657-665$.

[20] NENOT J.C. Métabolisme et toxicité du neptunium. In. : GALLE P. Toxiques nucléaires. Paris: Masson, 1982,210-216.

[21] NISHITA H., WALLACE A., ROMNEY E.M., SCHULZ R. Effect of soil type on the extractability of ${ }^{237} \mathrm{~Np},{ }^{239} \mathrm{Pu},{ }^{241} \mathrm{Am}$ and ${ }^{244} \mathrm{Cm}$ as a function of $\mathrm{pH}$. Soil Sci., $1981,132,25-34$

[22] NISHITA H., WALLACE A., ROMNEY E.M., KINNEAR J. Relationship between the chemical extractibility of several transuranic elements from soils and their uptake by wheat plants. Soil Sci., 1981, 132,60-65.

[23] OCDE/AEN. Comportement mésologique et biologique du plutonium et de certains autres éléments transuraniens. Paris: OCDE, 1981.

[24] PAGĖS M. Neptunium. In : Traité de Chimie Minérale (PASCAL P., Dir.). Paris : Masson, 1962, 15, 3' fasc., 237-324.

[25] PENTREATH R.J. The use of isotopic ratios in determiming the relative biological availabilities of transuranium elements. In : AIEA.- Techniques for identifying transuranium speciation in aquatic environment, Ispra, March 1980. Vienne: AIEA, 1981. 141-149.

[26] RANÇON D., ROCHON J. Rétention des radionucléides à vie longue par divers matériaux naturels. In : OCDE et CEE.- La migration des radionucléides à vie longue dans la géosphère (Bruxelles, 29-31 janvier 1979). Paris : OCDE, 1979, 301-322.

[27] ROBINSON A.V., GARLAND T.R., DRUCKER H., WILDUNG R.E. Plutonium resistant fungi and actinomycetes in soil : II. Alteration of plutonium form. In : BNWL-1950 Pt.II, 1976, 28-30.

[28] ROCHON J., RANÇON D., GOURMEL J.P. Recherche en laboratoire sur la rétention et le transfert de produits de fission et de transuraniens dans les milieux poreux. In : AIEA. - Underground disposal of radioactive wastes, Otaniemi, 2-6 Juil. 1979. Vienne: AIEA, vol. 2, 271-314.

[29] ROMNEY E.M., WALLACE A., SCHULZ R.K., KINNEAR J., WOOD R.A. Plant uptake of ${ }^{237} \mathrm{~Np},{ }^{239} \cdot 240 \mathrm{Pu},{ }^{241} \mathrm{Am}$ and ${ }^{244} \mathrm{Cm}$ from soils representing major food production areas of the United States. Soil Sci., 1981, 132, 40-59.

[30] ROSS B., KOPLIK C.M., GIUFFRE M.S., HODGIN S.P. A computer model of long term hazards from waste repositories. Radioact. Waste Manag., 1981, 1, 322-335.

[31] ROUTSON R.C., JANSEN G., ROBINSON A.V. ${ }^{241} \mathrm{Am},{ }^{237} \mathrm{~Np}$ and ${ }^{99} \mathrm{Tc}$ sorption on two United States subsoils from differing weathering intensity areas. Health Phys., 1977, 33, 311-317.

[32] SCHNEIDERMAN G.S., GARLAND T.R., DRUCKER H., WILDUNG R.E. Pu resistant fungi and actinomycetes in soil. In : BNWL-1950, Pt. 2, 1975, 25-28.

[33] SCHRECKHISE R.G., CLINE J.F. Comparative uptake and distribution of plutonium, americium and neptunium in four plant species. In : Transuranic elements in the environment (HANSON W.C., Ed) DOE/TIC-22800, 1980, 361-370.

[34] SCHULZ R.K. Root uptake of transuranium elements. In : NVO-178 (WHITE M.G., DUNAWAY P.B., Ed). 1977, 321-330.

[35] SCHULZ R.K., RUGGIERI M.R. Uptake and translocation of neptunium-237, plutonium-238, plutonium 239, 240, americium-241 and curium-244 by a wheat. Soil Sci., 1981, 132, 77-82.

[36] SULLIVAN M.F., CROSBY A.I. Absorption of ${ }^{233} \mathrm{U},{ }^{237} \mathrm{~Np},{ }^{238} \mathrm{Pu},{ }^{244} \mathrm{Cm}$ and ${ }^{253} \mathrm{Es}$ from the gastrointestinal tract of newborn and adult rats. In : BNWL-1950, Part.1, $1975,105-108$. 


\section{R. BITTEL}

[37] SULLIVAN M.F. Actinide absorption from gastrointestinal tract. In : Actinides in man and animals (WRENN M.E., Ed) Salt Lake City : R.D. Press, 1981, 311-322.

[38] SULLIVAN M.F., MILLER B.M., RYAN J.L. Effect of mass on gastrointestinal absorption of neptunium. In : PNL-4100, Part 1, 1980, 119-122.

[39] THOMPSON R.C. Neptunium, the neglected actinide : A review of the biological and environmental literature. Radiat. Res., 1982, 90, 1-32.

[40] WILDUNG R.E., GARLAND T.R. The relationship of microbial processes to the fate and behaviour of transuranic elements in soils, plants and animals. In : Transuranic elements in the environment (HANSON W.C., Ed). DOE/TIC - 22800, 1980, 300-335.

[41] ZAJIC J.E. Microbial biogeochemistry. New-York: Academic Press, 1969. 